\title{
Psychometric functions and a correlation model of binaural detection*
}

\author{
ELI OSMAN, PAUL N. SCHACKNOW, and PI-FONC LIN TZUO \\ Department of Psychology, Brooklyn College, CUNY, Brooklyn, New York 11210
}

\begin{abstract}
Sets of psychometric functions for the detection of tone in noise are reexamined for several interaural listening conditions. Best-fit sets of functions are determined for a "correlation" model of binaural masking level differences (MLDs). Based on only three parameters, this model predicts both the forms and relative positions of all such functions. The finding that MLDs are relatively independent of the detection level chosen for their determination is predicted by the model, and the sets of theoretical functions describe the data nearly as well as a previously determined empirical relation which requires $M+1$ parameters for $M$ curves.
\end{abstract}

The form of the psychometric function for the detection of tonal signals in noise was analyzed for several interaural listening conditions, with varying interaural relations both for signal and for noise, by Egan, Lindner, and McFadden (1969). Expressed in decibels, comparison of the signal-to-noise ratios for a fixed level of detection for two different listening conditions is the masking level difference (MLD) between these two conditions. Analysis of the psychometric functions is important for at least two reasons. First, differences in form as well as position of these functions for different listening conditions would require explanation by, and thus provide further test of, the descriptive power of existing models of binaural detection. Second, it is useful for future experimental work to determine whether the MLD is dependent on the detection level chosen for its determination.

Egan, Lindner, and McFadden show that the data are approximately described by the empirical relation

$$
d^{\prime}=m\left(E / N_{o}\right)^{k}
$$

where $\mathrm{m}$ and $\mathrm{k}$ are constants for a particular function, $\mathrm{E}$ is the energy of the signal, $N_{o}$ is the noise power per unit bandwidth, and the criterion-free measure $\mathrm{d}^{\prime}$ is readily $t$ ransformed into maximum percent correct by consulting a table for the area under the normal density function corresponding to $d^{\prime} / 2$. Given that the results suggested that $\mathrm{k}$ could be considered constant for a given listener and that only $m$ depends on the interaural condition, the MLD is independent of the level of performance chosen for its definition. When the psychometric functions are plotted as percent correct vs $10 \log \left(E / N_{o}\right)$, they are approximately parallel, so that the horizontal distance between any pair is their relative MLD.

The $\mathrm{d}^{\prime}$ equation (Eq. 1) used to describe the

* This work was supported by Grant No. 1 R03 MH20237-01 MSM from the U.S. Department of Health, Education and Welfare. Public Health Service, and by Grant 1719 from the City University of New York Faculty Research Award Program (both to E. Osman). psychometric functions is a purely empirical relation that has no predictive power. It requires, in general, two free parameters for each psychometric function. However, empirical results suggest that a set of such functions representing the results of a given listener or average results of a set of listeners may require only a single value of the parameter $\mathrm{k}$ and, in addition, a different value of $\mathrm{m}$ for each curve. There are some models of the monaural detection process that predict both the form and location of the psychometric function. One of these is the monaural energy detector (Green \& Swets, 1966), which yields a psychometric function that is approximately $\mathrm{d}^{\prime}$ with $\mathrm{k}=1 .{ }^{1}$ Several models of the binaural detection process which propose to explain MLDs have appeared in the literature, and currently only one of these, the correlation $(\rho)$ model (Osman, 1971), which may be regarded as a binaural analogue of the energy detector, provides explicit equations for the density functions conditional on noise alone and on noise plus signal. This model, using at most three free parameters, predicts both the form and relative location of each of a set of psychometric functions obtained under many interaural listening conditions. The purpose of this paper is to compare best-fit theoretical psychometric functions based on the correlation model to both data and best-fit empirical functions as presented in Egan, Lindner, and McFadden (1969).

The correlation model has so far been used to describe MLD data from a variety of experiments (Osman, 1971; Osman, in press). These include studies of antiphasic, homophasic, and monaural input configurations at different signal frequencies, level of detectability for tones in noise for monaural listening at different frequencies, varying interaural correlations of signal and of noise where the noise correlation is generated either statistically by mixing the outputs of independent noise generators or deterministically by varying interaural time shifts or phase shifts. and simultaneous variation of interaural amplitude ratio and phase shift for signal.

The correlation model assumes that the receiver computes a decision quantity. D. which is a weighted 
sum of the energies in each of the two auditory channels and the interchannel cross correlation, after internal noise is added to each input and the results are appropriately filtered. The three free parameters of the model are: $\mathbf{R}=$ the ratio of internal to external noise power densities, $\rho_{Y}=$ the normalized interchannel correlation coefficient for internal noise, $W=$ the effective rectangular bandwidth of resultant noise. The input parameters of interest are: $\rho_{\mathrm{N}}=$ the normalized interaural correlation coefficient for input noise, $\rho_{\mathbf{s}}=$ the normalized interaural correlation coefficient for signal, $\mathrm{T}=$ the duration of the observation interval, $\mathrm{E}=$ signal energy, $N_{0}=$ input noise power density. The quantities $W$ and $T$ always appear as the product $W T$, and this is treated as a single parameter in place of its factors.

For the data of Egan, Lindner, and McFadden, the continuous white noise (bandlimited by the earphones) was at a constant spectrum level of $45 \mathrm{~dB}$ re 0.0002 microbar. Under these restricted conditions of fixed and equated noise levels at the two auditory channels (each channel is identified below by the subscript $i$, where $i=1,2$ ), the equations of the correlation model are somewhat simplified and derive from

$$
\mathrm{D}=\int_{0}^{\mathrm{T}} \mathrm{dt}\left[\mathrm{x}_{1}^{2}(\mathrm{t})+\mathrm{x}_{2}^{2}(\mathrm{t})-2 \rho_{\mathrm{n}} \mathrm{x}_{1}(\mathrm{t}) \mathrm{x}_{2}(\mathrm{t})\right] /\left(1-\rho_{\mathrm{n}}^{2}\right)
$$

where each $x_{i}(t)$ is the resultant waveform in Channel $i$, and $\rho_{\mathrm{n}}$ is the effective normalized interchannel correlation coefficient, which can be expressed as

$$
\rho_{\mathrm{n}}=\left(\rho_{\mathrm{N}}+\mathrm{R} \rho_{\mathrm{Y}}\right) /(1+\mathrm{R}) .
$$

The distribution of $\mathrm{D}$ is approximately Gaussian, and the conditional means and variances are given in Eqs. 4, 5, and 6 for the experimental conditions of interest in this paper.
The Egan, Lindner, and McFadden data were obtained in two-interval forced-choice (2IFC) and single-interval forced-choice or yes-no (Y-N) tasks, with equal a priori probabilities of noise alone and noise plus signal for each observation interval. For the correlation model in the 2IFC task

$$
\text { maximum percent correct }=\int_{-\mathrm{h}}^{\infty} \mathrm{dz} \varphi(\mathrm{z}),
$$

where $\varphi$ is the Gaussian density function, and

$$
\mathrm{h}=\left(\mu_{\mathrm{n}+\mathrm{s}}-\mu_{\mathrm{n}}\right) / \sqrt{\sigma_{\mathrm{n}+\mathrm{s}}^{2}+\sigma_{\mathrm{n}}^{2}} .
$$

For the correlation model in the $\mathrm{Y}-\mathrm{N}$ task

maximum percent correct

$$
=1 / 2\left\{\int_{z_{a_{n}}}^{z_{b_{n}}} d z \varphi(z)+1-\int_{z_{a_{n}+s}}^{z_{b_{n}}+s} d z \varphi(z)\right\},
$$

with

$$
\begin{aligned}
& \mathrm{z}_{\mathrm{a}_{\mathrm{n}}}=-\left(\mu_{\mathrm{n}+\mathrm{s}}-\mu_{\mathrm{n}}\right) /\left(\sigma_{\mathrm{n}+\mathrm{s}}-\sigma_{\mathrm{n}}\right), \\
& z_{b_{n}}=\left(\mu_{n+s}-\mu_{n}\right) /\left(\sigma_{n+s}+\sigma_{n}\right), \\
& z_{a_{n+s}}=-\left(\mu_{n+s}-\mu_{n}\right) /\left(\sigma_{n+s}-\sigma_{n}\right), \\
& z_{b_{n+s}}=-\left(\mu_{n+s}-\mu_{n}\right) /\left(\sigma_{n+s}+\sigma_{n}\right) .
\end{aligned}
$$

Each of Figs. 1 and 2 reproduces the data presented by Egan, Lindner, and McFadden (1969). Also shown in each figure is the least squares best-fit set of curves based on the correlation model. The best-fit parameters were obtained with the aid of a computer using a direct search technique. Figure 1 is for five interaural conditions in a 2IFC task. Figure 2 is for three interaural conditions in a

\footnotetext{
Experimental Condition

Noise Alone

$\mathrm{N}_{\mathrm{o}_{1}}=\mathrm{N}_{\mathrm{O}_{2}}=\mathrm{N}_{\mathrm{o}}$

$\mu_{\mathrm{n}}=2 \mathrm{~N}_{\mathrm{o}}(\mathrm{R}+1) \mathrm{WT}$

$E_{1}=E_{2}=E$

$\sigma_{\mathrm{n}}^{2}=2 \mathrm{~N}_{\mathrm{o}}^{2}(\mathrm{R}+1)^{2} \mathrm{WT}$

$\mathrm{N}_{\mathrm{o}_{1}}=\mathrm{N}_{\mathrm{o}_{2}}=\mathrm{N}_{\mathrm{o}}$

$\mu_{\mathrm{n}}=2 \mathrm{~N}_{\mathrm{o}}(\mathrm{R}+1) \mathrm{WT}$

$E_{1}=E, E_{2}=0$

$\sigma_{\mathrm{n}}^{2}=2 \mathrm{~N}_{\mathrm{o}}^{2}(\mathrm{R}+1)^{2} \mathrm{WT}$

$\mathrm{N}_{\mathrm{o}_{1}}=\mathrm{N}_{\mathrm{o}}, \mathrm{N}_{\mathrm{o}_{2}}=0$

$\mu_{\mathrm{n}}=\mathrm{N}_{\mathrm{o}}(\mathrm{R}+1) \mathrm{WT}$

$\mathrm{E}_{1}=\mathrm{E}, \mathrm{E}_{2}=0$

$\sigma_{\mathrm{n}}^{2}=\mathrm{N}_{\mathrm{o}}^{2}(\mathrm{R}+1)^{2} \mathrm{WT}$

Noise Plus Signal

$$
\begin{aligned}
& \mu_{\mathrm{n}+\mathrm{s}}=2 \mathrm{~N}_{\mathrm{o}}(\mathrm{R}+1) \mathrm{WT}+2 \mathrm{E}\left(1-\rho_{\mathrm{s}} \rho_{\mathrm{n}}\right) /\left(1-\rho_{\mathrm{n}}^{2}\right) \\
& \sigma_{\mathrm{n}+\mathrm{s}}^{2}=2 \mathrm{~N}_{\mathrm{o}}^{2}(\mathrm{R}+1)^{2} \mathrm{WT}+4 \mathrm{~N}_{\mathrm{o}}(\mathrm{R}+1) \mathrm{E}\left(1-\rho_{\mathrm{s}} \rho_{\mathrm{n}}\right) /\left(1-\rho_{\mathrm{n}}^{2}\right) \\
& \mu_{\mathrm{n}+\mathrm{s}}=2 \mathrm{~N}_{\mathrm{o}}(\mathrm{R}+1) \mathrm{WT}+\mathrm{E} /\left(1-\rho_{\mathrm{n}}^{2}\right) \\
& \sigma_{\mathrm{n}+\mathrm{s}}^{2}=2 \mathrm{~N}_{\mathrm{o}}^{2}(\mathrm{R}+1)^{2} \mathrm{WT}+2 \mathrm{~N}_{\mathrm{o}}(\mathrm{R}+1) \mathrm{E} /\left(1-\rho_{\mathrm{n}}^{2}\right) \\
& \mu_{\mathrm{n}+\mathrm{s}}=\mathrm{N}_{\mathrm{o}}(\mathrm{R}+1) \mathrm{WT}+\mathrm{E} \\
& \sigma_{\mathrm{n}+\mathrm{s}}^{2}=\mathrm{N}_{\mathrm{o}}^{2}(\mathrm{R}+1)^{2} \mathrm{WT}+2 \mathrm{~N}_{\mathrm{o}}(\mathrm{R}+1) \mathrm{E}
\end{aligned}
$$$$
\mu_{\mathrm{n}+\mathrm{s}}=2 \mathrm{~N}_{\mathrm{o}}(\mathrm{R}+1) \mathrm{WT}+\mathrm{E} /\left(1-\rho_{\mathrm{n}}^{2}\right)
$$$$
\sigma_{n+s}^{2}=2 N_{o}^{2}(R+1)^{2} W T+2 N_{o}(R+1) E /\left(1-\rho_{n}^{2}\right)
$$$$
\mu_{\mathrm{n}+\mathrm{s}}=\mathrm{N}_{\mathrm{o}}(\mathrm{R}+1) \mathrm{WT}+\mathrm{E}
$$ 
$\mathrm{Y}-\mathrm{N}$ task. For each figure, the data set is the average over a fixed group of listeners. For the notation used, $\mathrm{N}$ denotes noise, $S$ denotes signal, $O$ denotes a 0 -deg interaural phase shift, $\pi$ denotes a 180-deg interaural phase shift, $U$ denotes uncorrelated inputs, and $\mathrm{m}$ denotes monaural presentation. In terms of interaural correlation, $\mathrm{NO}$ means $\rho_{\mathrm{N}}=+1$, NU means $\rho_{\mathrm{N}}=0, \mathrm{~N} \pi$ means $\rho_{\mathrm{N}}=-1$, SO means $\rho_{\mathrm{s}}=+1$, S $\pi$ means $\rho_{\mathrm{s}}=-1$, and $\mathrm{Nm}$ and $\mathrm{Sm}$ represent monaural inputs for noise and for signal, respectively.

The set of correlation model equations predicts that psychometric functions in both 2IFC and $\mathrm{Y}-\mathrm{N}$ experiments for all interaural conditions where noise levels are equated at the two ears are parallel to each other in graphs of percent correct vs $10 \log \left(\mathrm{E} / \mathrm{N}_{\mathrm{o}}\right)$. The MLD for any two such conditions is predicted to be independent of the level of detection used for its measurement. The sets of least squares correlation model curves of Figs. 1 and 2 were obtained by assuming that the receiver operation is given by the expression for $D$ (Eq. 2) for all interaural conditions. Note that the theoretical curve for $\mathrm{NmSm}$ is displaced slightly to the left of, and is slightly nonparallel to, the theoretical curve for NOSO. The relative positions of these two curves are fixed in the model at K by choice of WT in the equation

$$
K=10 \log \left[\left(h+\sqrt{h^{2}+2 W T}\right) /\left(h+\sqrt{h^{2}+4 W T}\right)\right] .
$$

The data do not suggest a comparable shift of $\mathrm{NmSm}$ relative to NOSO. At least three assumptions can be

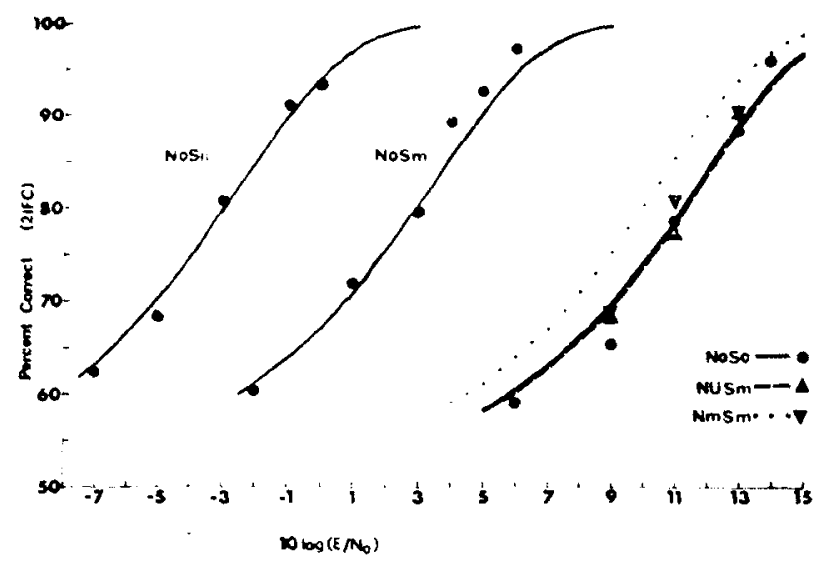

Fig. 1. Average psychometric functions for five interaural conditions. Each point is based on data collected simultaneously from four listeners on 180 or 240 trials in a 2IFC task. The signal was a $500-\mathrm{Hz}$ tone of $250 \mathrm{msec}$ duration. Noise was continuous at a spectrum level of $45 \mathrm{~dB}$ re 0.0002 microbar. The "best-fit" set of five solid curves is based on the correlation model with $R=0.18, \rho_{Y}=0.51$, and $W T=41$. These values predict that, relative to NOSO, the MLDs are $14.1 \mathrm{~dB}$ for $\mathrm{NOS} \pi$, $8.3 \mathrm{~dB}$ for NOSm, $-0.1 \mathrm{~dB}$ for NUSm, and from 0 to $1.6 \mathrm{~dB}$ for $\mathrm{NmSm}$ (where the value of $\mathrm{NmSm}$ depends on percent correct). The data are from Fig. 5 of Egan, Lindner, and McFadden (1969).

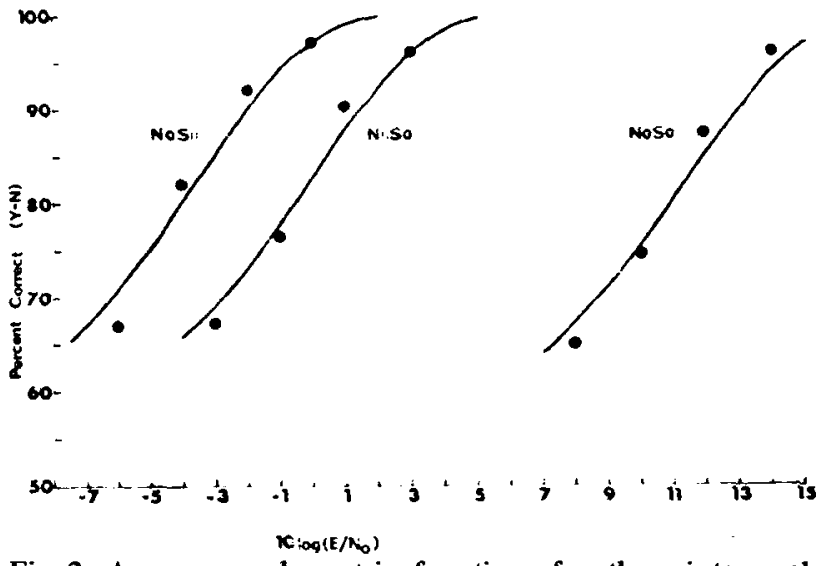

Fig. 2. Average psychometric functions for three interaural conditions. Each point is based on data obtained from three listeners on $\mathbf{4 8 0}$ trials on a $Y-N$ task. The signal was a $400-\mathrm{Hz}$ tone of $125 \mathrm{msec}$ duration. Noise was continuous at a spectrum level of $45 \mathrm{~dB}$ re 0.0002 microbar. The "best-fit" set of three solid curves is based on the correlation model with $R=0.11, \rho_{Y}$ $=0.38$, and $W T=29$. These values predict that, relative to NOSO, the MLDs are $15.0 \mathrm{~dB}$ for $N O S \pi$, and $11.5 \mathrm{~dB}$ for $\mathrm{N} \pi \mathrm{SO}$. The data are from Fig. 10 of Egan, Lindner, and McFadden (1969).

made to explain this. First, the internal noise parameters may be different if external noise is presented to only one ear. However, to fully explain the shift observed, the internal noise in the nonsignal ear may have to be unreasonably large. ${ }^{2}$ Second, the effective bandwidth after binaural noise inputs may be less than that after monaural inputs; a factor of two would imply that $\mathrm{K}=$ $0 \mathrm{~dB}$, and predict coincidence of the curves for NOSO and $\mathrm{NmSm}^{3}$ Third, the listener may utilize the information in only one channel whenever this will produce even a small advantage in detectability. This third assumption, as well as the first or second, does not change the nature of the detection process in the model. It says only that two of the three terms of $D$ may be disregarded (weighted by zero).

For the set of functions in Fig. 1, the root mean square deviation of data points from theoretical points is .027 , with $\left(\mathrm{R}, \rho_{\mathrm{Y}}, \mathrm{WT}\right)=(.18, .51,41)$. For the set of functions in Fig. 2, the root mean square deviation of data points from theoretical points is .021 , with $\left(\mathrm{R} \rho_{\mathrm{Y}}, \mathrm{WT}\right)=(.11, .38,29)$. Using the Egan, Lindner, and McFadden values of $\mathrm{k}$ for their $\mathrm{d}^{\prime}$ equations, we computed the comparable root mean square deviations for their psychometric functions (which appear in their Figs. 5 and 10). The resulting values are .016 for the data of Fig. 1 and .017 for the data of Fig. 2. The set of best-fit correlation model functions was also obtained for the data of Fig. 1 by assuming that NOSO and NUSm are in effect NmSm conditions. In this case, the fit is visually good, since the three curves on the right are replaced by one, and the root mean square deviation becomes 019 , with $\left(\mathrm{R}, \rho_{\mathrm{Y}}, \mathrm{WT}\right)=(.09, .32,88) .{ }^{5}$ Thus. except for the displacement of the $\mathrm{NmSm}$ curve in Fig. 1. which can be corrected by a reasonable assumption, the best-fit sets of curves for the correlation 
model describe the data very well, and it would clearly be difficult to argue that the functions based on the $\mathrm{d}^{\prime}$ equation (Eq. 1) are to be preferred. Note also that each set of values of the parameters $R, \rho_{\mathbf{Y}}$, and WT required to fit the Egan, Lindner, and McFadden data displayed in Figs. 1 and 2 is in reasonable agreement with those previously estimated for the correlation model (Osman, 1971); and the values of $W$, calculated using the duration of the observation interval as set by the experimenter for $\mathrm{T}$, are in reasonable agreement with values determined in the literature (Green \& Swets, 1966).

Since the correlation model has successfully described the MLD (based on a fixed level of detection) for many interaural conditions obtained by changing the values of $\rho_{\mathrm{S}}$ and $\rho_{\mathrm{N}}$, and since it now can be expected that all of the corresponding psychometric functions would be parallel, it is relevant that the predicted set of correlation model psychometric functions would still be based on only three parameters, while empirical fits using the $\mathrm{d}^{\prime}$ equation would require $M+1$ parameters, where $\mathbf{M}$ is the number of functions to be described.

\section{REFERENCES}

Egan. J. P., Lindner, W. A., \& McFadden. D. Masking-level differences and the form of the psychometric function. Perception \& Psychophysics, 1969. 6, 209-215.

Green, D. M. Interaural phase effects in the masking of signals of different durations. Journal of the Acoustical Society of America, 1966, 39, 720-724.

Green, D. M., \& Swets, J. A. Signal detection theory and psychophysics. New York: Wiley, 1966.

Osman, E. A correlation model of binaural masking level differences. Journal of the Acoustical Society of America, $1971,50,1494-1511$.

Osman, E. Correlation model of binaural detection: Interaural amplitude ratio and phase variation for signal. Journal of the Acoustical Society of America, in press.

\section{NOTES}

1. Green (1966) has used this to fit data obtained under each of several interaural listening conditions.

2. For the $\mathrm{NmSm}$ condition with presumed internal noise in the channel for the nonsignal ear, Eqs. 35.38 of Osman (1971) yield

$$
\mathrm{h}=\frac{\left(\frac{E}{\mathrm{~N}_{\mathrm{o}}}\right)\left(\frac{1}{1+\mathrm{R}}\right)\left(1+\frac{\rho_{\mathrm{n}}^{2} \mathrm{R} \mathrm{R}_{\mathrm{Y}}}{1+\mathrm{R}}-\rho_{\mathrm{n}}^{2}\right) /\left(1-\rho_{\mathrm{n}}^{2}\right)}{\sqrt{2 \mathrm{WT}\left(1+\frac{\mathrm{R}^{2} \mathrm{R}_{\mathrm{Y}}^{2}}{(1+\mathrm{R})^{2}}\right)+\left(\frac{\mathrm{E}}{\mathrm{N}_{\mathrm{o}}}\right)\left(\frac{2}{1+\mathrm{R}}\right)\left(1+\frac{\rho_{\mathrm{n}}^{2} \mathrm{R}^{2} \mathrm{R}_{\mathrm{Y}}^{2}}{(1+\mathrm{R})^{2}}-\rho_{\mathrm{n}}^{2}\right) /\left(1-\rho_{\mathrm{n}}^{2}\right)}},
$$

where it can be shown that $\rho_{n}=\rho_{Y} \sqrt{R /(1+R)}$, with $R$ now representing the ratio of internal to external noise power densities for the signal ear channel, and $R_{Y}$ defined as the ratio of internal noise power density in the nonsignal channel to internal noise power density in the signal channel. Utilizing the best-fit parameters of Fig. 1 (thereby not altering the psychometric functions for the nonmonaural conditions), psychometric functions were determined for the $\mathrm{NmSm}$ condition for various amounts of assumed internal noise in the nonsignal channel. For fixed $E / N_{0}$, with $0<R_{Y} \leqslant 1$ the variation in $h$ appears in the third decimal place, and consequently has a negligible effect on percent correct. This is also true if $\rho_{Y}$ is increased to unity. Thus, both the position and slope of the $\mathrm{NmSm}$ psychometric function are virtually constant for nonzero values of $R_{Y} \leqslant 1$. These functions are approximately parallel to those shown in Fig. 1, and $10 \log \left(E / N_{0}\right)$ equal to slightly more than $9 \mathrm{~dB}$ will yield $76 \%$ correct decisions for any of them. Thus, for internal noise levels in the nonsignal ear channel which do not exceed the internal noise level of the signal channel, the $\mathrm{NmSm}$ curve is displaced only slightly to the right of its position in Fig. 1 .

3 . In the numerator of the argument of the logarithm of the expression for $\mathrm{K}, \mathrm{W}$ represents the bandwidth under monaural listening; and in the denominator, it represents the bandwidth under binaural listening.

4. Here we would have a new set of best-fit parameters which would yield a single $\mathrm{NmSm}$ curve to fit the data for $\mathrm{NmSm}$, NOSO, and NUSm. The theoretical functions for NOSO and NUSm based on binaural processing would then be displaced to the right of the data points. However, they would not be relevant, since the assumption is specifically that NOSO and NUSm inputs are processed monaurally-effectively as $\mathrm{NmSm}$. Generally, it may now have to be assumed that the receiver can adjust the relative weights assigned to the two energy terms and one cross-correlation term in the computation of the decision variable, $\mathrm{D}$, in order to improve performance.

5 . The sum of squares deviations of data points about their mean is . 323 for Fig. 1 and .164 for Fig. 2. Thus, in eacit case, the set of psychometric functions used to fit the data accounts for more than $99 \%$ of the variance.

(Received for publication January 3, 1973; revision received May 14, 1973.) 